DYEING, BLEACHING, PRINTING, FINISHING,

THE BLEACHING OF WOOL WITHOUT SULPHUR.

ORDINARILY wool is deprived of its yellow or gravis
tinge by the application of sulphur in different ways, but mostly in the form of sulphurous anhydride, the pungent gas given rise to by the combustion of sulphur in air; this sired whiteness it is necessary to drown the remaining yel
lowing shade by the application of bluing, either with in digo carmine, indigo purple, aniline blue, aniline violet, ultramarine, Prussian blue, or similar substances. This, as a rule, gives satisfactory results as far as the appearance of
the cloth goes, but the substances used undergo, more or less, a change through the action of the sun and soap when A German dyer, named

A German dyer, named Kallab, bas recently invented tibers, can be bleached and blued in such a manner that the shade withstands milling, washing, and the action of sunthen brought, still damp, into a bath of pure water of or-
dinary temperalure, to which has been added a very small quantity of finely powdered purple indigo, say 40 to 90 grains the water, when placed into a white earthenware vessel, bas
only a faint bluish tinge. The cloth is left a short time in this bath, during which the finely divided indigo deposits
up:n it; it is then wrung and brought into the bleaching

The bleaching liquor is contained in a wooden well-closed vessel, and consists of a solution of freshly prepared sub-
sulphate of soda $\left(\mathrm{NaHSO}_{3}\right.$ ) of 1.0069 to 1.0283 specific gravity, according as there is a necessity for more or less three to six cubic inches of acetic acid of 50 per cent., and free from any mineral acid. the whole is then well stirred,
and the yarn or cloth to be bleached introduced, after which the vessel is closed to prevent the atmospheric air entering. is converted into indigo-white, which latter is absorbed by
the fiber, while the sulphurous acid, formed from the byposulphite, performs the bleaching action. It takes from a
few to twelve and twenty-four hours for this operation when completed the cloth, taken out of the bath, must show
a clear white, with a bluish tinge, after baving been rinsed in water. As soon as this is attained the cloth is all taken
out, drained, and exposed to the action of the air, by which means the indigo is again brought back to a blue color, and
gives the cloth a bluish shade. Where the bath has been strong one, it is advisable to treat the cloth afterward in a
solution - of crystals of soda of one half to one per cent. Finally the cloth or yarn is washed in clear running water. wrung and dried in the air, or a stove at a temperature of
$1\left(10^{\circ}\right.$ to $110^{\circ}$. The foregoing proceeding may be varied by adding the acetic acid after the bleaching bath, but this dehas to retain the indigo-white in process of formation on the fiber, but also to prevent that the blue indigo, which at the beginning adberes only mechanically to the cloth, does acid can be easily tested; when indigo, ground damp, is put
into a vessel containing water, the latter will for days ap. pear blue; as soon, When bleaching loose wool it is best to add the indigo to
the hyposulphite bath, to place the wool in after a quarter
of an hour, and half an hour later to add slowly the neces. sary quantity of acetic acid (one part acid to ten parts
water) without taking the wool out. The remainder of the operation is as above.
If after the drying the cloth does not show sufficient whiteness, it is advisable to submit it to a second bleaching without any further addition of indigo, for which an old
bath can be used. It is in that case necessary to first test the bath with nitric acid, to find out if it separates sulphur. If this action occur, it is a proof that the old bath still con-
tains subsulphuric acid; in the contrary case it is necessary to add $1-10$ th or $1 / 4$ of the previously added hyposulphite,
and to leave the cloth in the bath until the hyposulphite has changed to sulphite of soda; the cloth is then taken out,
and so much nitric acid added until the presence of sulphurous acid is easily detected by the smell, and the cloth then
reintroduced until it is completely bleached. The first submersion accomplishes the fixation of the indigo on the cloth the second corresponds with the old method of bleaching bleached a second time, the bath can be made of a specific gravity of 1.035 to 1.013 , and without addition of acetic acid. Such a bath can be regenerated by the addition of ni-
tric acid until it smells of sulphurous acid, and then the addition of metallic zinc.

subsulphate of lime in place yellow, it is advisable to tak subsulphate of lime in place of the salts of potash or soda.
The former are obtained by adding metallic zinc to a solution of bisulphite of potash; the vessel is then closed, and
occasionally shaken; after an hour the clear liquid is poured off (and which contains a little zinc in the form of a double
salt of zinc and sulphide of potash), and used for the bleachsalt of zinc and sulphide of potash), and used for the bleach-
ing bath. To be able to bleach with byposulphite of calciliquid, until it reacts slightly alkaline. After this a bath of byposulphate of calcium is prepared without any further add the wool added without being previously of 1 to with in-
and
dion digo. The wool is then washed,
acid, and washed a second time.

When it is desirable to have a white with a greenish tinge the cloth is passed through a water-bath slightly acidulated
with nitric or sulphuric acid. Where a reddish or violet shade of the white is desirable, the cloth is passed through shade of the white is desirable, the cloth is passed through
a bath slightly colored to the required tinge.
The strength of the baths mentioned depends upon the degree of color to be removed, and as a rule is taken weaker

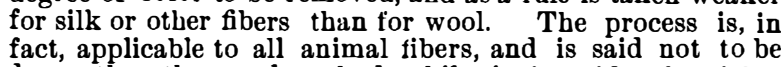
fact, applicable to all animal fibers, and is said not to be

ON AN ANILINE BLACK WHICH DOEB NOT TURN GREEN.

I WrSH to call the attention of the Society to a particular
fact in the history of aniline black. Without entering into details, I will say that my dyeing processes consist essen-
tially in causing salts or metallic oxidizing acids to act upon tially in causing salts or metallic oxidizing acids to act upon
aniline in an acid solution in presence of the goods to be
dyed. These become green, darker and darker, and finally turn black when the oxidation reaches a sufficient point, and
the flot remains perfectly limpid.
Besides the novelty of this kind of dyeing, I wish to point novelty of this kind of dyeing, I wish to point constitution of these blacks and which is quite different
from its mode of action in Lightfoot's process. In this later process the metal has no other effect than simply decom The lower oxides of chlorine, which produce a black. point, and explain perfectly bow it is that infinitely small
doses of copper, and especially of vanadium, suffice to produce the color.

In my blacks, on the contrary, it is remarked that in spit of the acidity of the solutions, the metallic oxides derived
from the salts or the acids employed fix themselves upon the much more intimate than that of ordinary lake colors, and The blacks that I produce will be blacks of metal-anile
Thers One or more equivalents of hydrogen are replaced by one of ulterior oxidation a special stability because of the stagen. This is what I said textually in my patents of Novem-
ber 3d, 1874, and in the claim of my English patent of October 21st of the same year I insisted upon the fact that this
metallizing action of the metallic salts may either precede or succeed the oxidizing action.

[N. B.-Metallic oxides in contact with or anic matter ar

not necessarily stable. ]
I claimed at the same time the application of these metal-
anile blacks in printing, but I was stopped by the diffculty of reserving the whites or the other colors associated
coling with the black.
The receipts which have since been given are merely a particular application of my theory, a special determination
of the reagents pointed out by me, with the object of not of the reagents pointed out by me, with the object of not
soiling the whites and not destroying colors other than The authors of these receipts, to whom I bave imparted my theories and my results, have asserted that the black
did not turn green in consequence of a more complete oxi Two very simple experiments enable me to prove that this Let us take a piece with black and white strip

Let us take a piece with black and white stripes, printed
Lightfoot's process and subject to greening. We fix the black by taking it through a boiling beck of
cidified persulphate of iron. When the transformation is complete, and the black no longer turns green, we remark
that the beck no longer contains any ferric salt, and that all the oxide of iron which came from it may be found upon the black parts of the cloth, while the white
soiled if the proportion of acid was sufficient.

Thus, therefore, aniline black, in fixing itself, attracts iron portions of the cloih.

The following example is still more decisive, permitting us to distinguish two phases in the action of the reagent second a metallizing part. Wo may fix the black with
chromic acid, acidified with sulphuric acid. As soon as a chromic acid, acidified with sulphuric acid. As soon as a
piece printed with aniline black, capable of turning green, has been worked for some minutes in a boiling beck con-
taining a certain proportion of this reagent, the chromic
acid is very rapidly reduced, and the liquid, which was yelacid is very rapidly reduced, and the liquid, which was yellow, becomes green. At this moment, though the reagent turn green if exposed to sulphuric acid. We may double,
triple, quadruple the proportion of the reagent, and may turn the black grayish, and finally destroy it, but still without hindering it from turning green,
If, on the contrary, we work the goods in the beck with the original lot of the reagent for thirty to forty-five minutes, the liquor turns from green to a violet red. At this
moment the black is fixed, and has become incapable of greening. It has attracted the total of the chromic oxide
detived from the reduction of thechromic acid, and of ohich there no longer exists a trace in the bath, but which may now of chrome remains in the bath, asks the Chemical Reviero, to what is the violet red color due? It is well known that cer-
tain modifications of the salts of chrome in solution display tain modificatio

To sum up, aniline black is capable of forming with certain metallic oxides true combinations which give it a pe-
culiar stability. This metallization of aniline may eithe precede or follow the oxidizing action which transforms it nto black. The combination of aniline and of the metal is a true substitution. Aniline black incapable of greening is ON CERIUM ANILINE BLACKS.

'I'HE application of cerium in the development of aniline ing. I take the liberty of communicating my observations. This application has more than a merely historical interest,
since cerium is a metal which, by its analytical properties, the iron group. All the other metals in use for aniline blacks, such as copper, vanadium, tungsten, are precipitable by sulphureted bydrogen or by sulphuret of ammonium as
sulphurets, while cerium is only precipitated as a bydrated The use of cerium for aniline blacks, like that of the other metals employed, is based upon its property of becoming
easily reduced and again oxidized. It forms two series of salts corresponding with its two stages of oxidation-salts of oxidized in presence of oxidizing agents, such, for instance, as the chlorates or chloric acid, forming the hydrated oxide of cerium of a red color. The salts of oxide of cerium, on he other band, are reduced by deoxidizing agents, such a
alcohol with muriatic acid, to salts of the suboxide.
It is in virtue of these properties that the chlorate em It is in virtue of these properties that the chlorate em
ployed in mixtures for aniline blacksis decomposed, and the
aniline forms black. The action continues till all the chlorate bas been decomposed

The chlorides and sulphates of cerium are soluble in
water; and in my first experiments I made use of the bisul hate of cerium - a salt of a yellow color, soluble in six part of water. I took 75 grains of bisulphate to $13 / 4$ pint of
color, weighing 2 lbs. $61 / 2$ ozs., and containing $23 / 4$ ozs. muriate of aniline. The color printed very well, and did no
ritor. att, ack the doctors. After printing it appeared of a very
lig it green, and then become of a deep green, after aging
for 24 hours at a temperature of 77 deg. Fabr. by the dry bulb thermometer, and 68 deg. Fabr. by the wet bulb or finally takes a rich velvety black.

But the bisulphate of cerium, as well as the chloride, is not to be found in the market, though the oxalate of cerium le in water. It is in a sufficiently fine powder to be mixed On taking, however, 75 grains to 1.75 pint of color an age-
with the cols. ing of 24 bours is insufficient, and more than a week must cause of this inconvenience, and I, therefore, treated the ox alate with dilute sulphuric acid in presence of alcohol. On

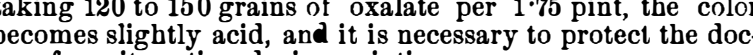
I rom its action during printing.

I bave used this color for chrome orange styles, on an ani-
ine black ground. When the black is oxidized I fixed the lead with a bath of sulphuric acid at 5 deg. B., and after I bave not tried if it is possible to succeed better by com-
. pletely dissolving the cerium in muriatic acid, then neutralizIn concluns a color easier to apply might be obtained. its use may seem, presents little advantage over the sulphu ret of copper. It is true that one-fourth part the weight of a salt of cerium gives a good result, but this reductlon is Consequently these salts have not found any particular prac-

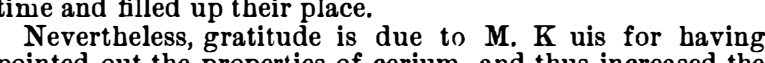
pointed out the properties of cerium, and thus increased the number of elementary bodies applicable in the formation of rial Society of Rouen, in Moniteur de la Teinture.

PAINTING UPON WOOD AND CANVAS-THE DETERIORATION OF OIL PAINTINGS,

OrL paintings are subject to various kinds of changes which may be considered as diseases, requiring different
treatment according to their different nature. A science treatment according to their different nature. A science
needs to be formed - a pathology and therapeutics of oil needs to be formed-a pathology and therapeutics of oil
paintings. The pathology would bave to describe and
explain those diseases and their progress, and to develop the methods by which a correct diagnosis could be arrived
th in each individual case. The therapeutics would teach at in each individual case. The therapeutics would teach
the remedies which might be applied either to cure or to the remedies which might be applied either to cure or to
alleviate the disease, or at least to stop its progress. A hyavoid pernicious influences, and which, besides, while giving precepts for the technical process of painting, would bave to
forestall those constitutional diseases which, even in cases where no noxious influences can be traced, are the causes of decay, after a comparatively short period of existence. As
medical science is above all things based on anatomy and physiology, so the exact knowledge of the structure of fits disease. Unfortunately, direct investigation alone can procure no such exact knowledge; on the contrary, we are he material as well as of the technical methods adopted by The excellent works of cennino Cennini, Mérimée, Sir Charles Eastlake, Mrs. Merrifield and others, have already
furnished most valuable material; but still the field for investigation remains unlimited; for, in order to enable us to secure the conservation of each valuable painting, we ought
to know exactly how it was made. The artists of the presnt time would spare infinite trouble to the investigators of future times if, along with their works, they would leave the
account of their practice in the case of each picture. A
treatment without exact knowledge of the normal condition, as well as of the nature of the disease, is, as we shall see,
s dangerous for the picture as it would be in the case of living beings.

Professional restorers of pictures admit this danger in a
eneral way; each of them, however, is convinced that he general way; each of them, however, is convinced that he
himself, by his personal knowledge, skill and care, knows
how to avoid it. The public pays too little attention to the subject, and therefore it occurred to me that it might be usetul to give a short account of what we know about this
question, of the changes to which oil paintings are ex.
posed, as well as of the means either to avoid or to cure We have to consider, first, the material on which the artist
them. We have to consider, first, the material on which the artis
bas painted, that is, as far as oil painting is concerned, prinSecondly, the priming.

Secondly, the priming, that is, the substance with which
the surface was prepared in order to be made fit for paintThirdly, the painting itself, that is, the pigments and vehicles used for it, and the liquids that were added during he painting, the mediums, meguilp, siccative, varnish, es-
Fontial oils, etc.
Fourthly, the coat or coats of varnish spread over the

The wood on which a picture has been painted may eithe
picture. warp, or get chinks in it, or become worm-eaten, or even
litogether rotten. Against warping, the remedy usually ap altogether rotten. Against warping, the remedy usually ap-
plied is moisture. If the panel is very thick it is first made somewhat thinner; then the back is moistened, and the pic-
ture is left to lie on its back for twelve to twenty-four bours fter which time it will be found to have bent straight. Of course this must not be continued longer than necessary,
otherwise the convex surface, instead of becoming plane, would become concave. When straight, the picture is kept
so by beads which have to be adapted in a particular way, so by beads which have to be adapted in a particular way, a and contraction of the wood.

in the wood are drawn together by inserting pieces wood of a special shape.
Sublimate solutions are employed to destroy worms. Trifling losses of substance are replaced by cement. Small portions of rotten wood, not extending too near the paint-
ng, are cut out and replaced by wedge-shaped pieces. If, panel, is rotten, the picture must be separated from it and
transferred to new wood, or rather to canvas. This was first tried by Hacquin in Paris, and was per

* Lecture delivered at the Royal Institute of Great Britain, 1878. 
upon one of Raphael's Madonnas, in the Gallery du Louvre, the old masters bad been in possession of colors, that is pig- of the trough; or the space between the condensers when and upon Sebastian del Piombo's " Resurrection of Lar ments, the knowledge of which bas been lost, and that this th now in the National Gallery. The process no longer appears accounts principally for the difference between the oil so very marvelous; it is generally executed in the following painting of the fifteenth and sixteenth centuries, on the one way:
First of all the surface of the picture is pasted over with Band, and that of the eighteenth and nineteenthon the other.
hist at mistak. We know perfectly well the gauze and paper; after that the wood is made straight by pigments used by the old masters; we possess the same, and moistening, or, if necessary, by making incisions with the a considerable number of new ones, good as well as bad, in
saw, into which cuneiform pieces of wood are driven. By addition. In using the expression of good and bad, I am saw, into which cuneiform pieces of wood are driven. By addition. In using the expression of good and bad, I am
means of a tenon saw the panel is to be sawn into little principally thinking of their durability. From this point means of a tenon saw the panel is to be sawn into little principally thinking of their durability. From this point
squares, which must be removed by a chisel, and in thisway of view the pigments can be placed under three headings: \begin{tabular}{l|c|c|} 
the thickness of the wood is reduced to half an inch; it is & 1 . Those that are durable in themselves, and also agree \\
then planed until it becomes no thicker than paper, and the well with the other pigments with which they bave to be
\end{tabular} rest is removed by means of a knife and with the fingers. The painting being thus severed from its basis, it can be
fixed on canvas, if the priming is sufficiently preserved. In fixed on canvas, if the priming is sufficiently preserved. In but when as when sufficiently isolated remain unaltered; the opposite case, a mixture made of chalk and glue, or color, or alter the others, or produce a reciprocal modifismoothed after being dry. This done, the new canvas has to be fixed upon it by means of a mixture of glue,varnish and turpentine, and the substance of the picture pressed tightly and evenly against it by means of warm irons.

In order to avoid deterioration, the most minute precepts bave been given for preparing the panel. It bas to be taken
from the best oak, or nut trees, or cedars. The wood is to
be cut into boards during the winter-time, and kept till autumn before being dried; it can then be prepared only in
the following spring, etc. It would certainly be preferable to give up wood panels altogether for large pictures, and only to think of means to make the canvas stronger. For
small pictures, panels offer certain advantages, and can be small pictures, panels offer certain advantages, and can be
more easily preserved from decay. more easily preserved from decay.
In the canvas we meet with the results of injuries or spontaneous decay. A rent may be mended by rags of
linen stuck at the back of the picture. Even a hole may be filled up by pieces taken from other decayed paintings. If the picture is considerably damaged, it will be best to line preferable to sacrifice it by pulling off the threads one by
one, after having secured the painting itself by pasting one, after baving secured the painting itself by pasting
paper on the front of it. This done, the painting is transferred to another canvas in the same way as those removed from wood.
There are different modes of priming, which mav be brought under two principal heads: the distemper and the
oil priming. 1. The canvas is distempered by a mixture of chalk or
oil priming. plaster and paste, or glue, which may be laid on raw, unwith glue or paste. Several coats of this mixture must be put on in succession, one being perfectly dry before the next can be applied. Many of the older oil paintings are painted
on such ground. It bas the advantage of being quicker prepared, of absorbing the excess of oil, of permitting the color
to enter into the priming, and to dry quicker, and, moreover, of containing a white absolutely innocuous to other colors.

The inconveniences, on the other hand, are: that it more
easily breaks, and under the influence of humidity separates from the canvas.
2 . The oil priming consists of several coats of oil color As each of these must be perfectly dry before the next is laid on, and as, moreover, time must be given to the whole to dry completely before painting upon, in order to avoid slower than the distemper. Nevertheless it is now generally adopted.
Rey, in France, has pointed out a process which is a com promise between the two methods; he begins by distemper-
ing, and after several coats of distemper, having dried on ing, and after several coats of distemper, having dried one
after the other, he puts a coat of oil, which, as it were after the other, be puts a coat of oil, which, as it were
changes the distempered ground into an oil color ground. With oil priming it is of importance that the principal quantities of yellow, black, or other colors. For a whole
century a school, that of Bologna, predominated in Italy, century a school, that of Bologna, predominated in Italy,
which abandoned this principle. During the second balf of which abandoned this principle. During the second half of
the seventeenth and the first half of the eightcenth century most of the Italian masters of other schools followed its example. Probably for the purpose of obtaining mor easily the desired effect of the chiaroscuro they painted on a
brownish red priming, which consisted of bolus mixed with umber. Not one of those pictures has kept its original col-
oring. Not only has the priming caused all the dark parts to grow much darker, but it bas destroyed, or nearly so, al the glazing, so that only those colors can be recognized show you numerous instances of this. for, on account of the extreme fertility of this school, there is little difficulty in
procuring pictures of masters of that time or of their

pupils.
Wood priming does not require the same elasticity as that of the canvas, which ought to be capable of being rolled.
Therefore the priming of the wood shows less variations. It starch, paste size or glue, and more or less thickly laid In some pictures of gifferent centuries we find, either between the wood and the priming, or between the priming
and the painting, canvas, and exceptionally even paper.

The diseases of the priming are not of a very complicated
nature. They manifest themselves principally in three dif nature. They manifest themselves principally in three dif
ferent ways: 1 , by cracks in the priming itself; 2 , by the severance of the priming from the painting; 3 , by the seve
ance of the priming from the wood or the canvas. The third disease is by far the most frequent, especially among pictures on canvas distempered with paste. If small piecesonly are scaling off or blistering, they are fixed again to the
ground by letting a solution of size pass between the deground by letting a solution of size pass between the de-
tached part and the canvas, and pressing both gently
together. If the deterioration extends over a considerable surface, the picture bas to be lined. While this is being
done, and while the gluing substance penetrates into the done, and while the gluing substance penetrates into th picture, the detached parts are pressed on again with slightl
heated irons. If the whole priming threatens to come off
it will be better to take the picture entirely from the pane it will be better to take the picture entirely from the panel
or canvas, and to transfer it to a new canvas. tioned points, and among them two pictures, one in oil taken off from canvas, the other in tempera, taken off from wood. Both of them, strange to say, have escaped destruction without having been transferred to a new canvas, and
without being covered with paper, as is usually done, before taking them off. They show you the painting by itself
from both sides. I have, of course, used every precaution in bringing them safely over from Florence, where I happened to discover them carefully stowed a way among heaps of old pictures.
We come now to the most important part of the picture, 3. Those which are so little durable that, even when iso-
lated from other pigments, the mere contact of the vehicle. the air, or the light, makes them in time fade, darken, or The old masters used, without reserve, only those belong. The old masters used, with categories. For those belong. ing to the first of these three categories. For those belong-
ing to the second they imposed on themselves certain limits
and precautions. Those belonging to the third they did not se at all.

That some of the modern masters bave not followed these principles is not owing to a lost secret, but to the fact that they disregarded those well known principles, and even con-
ciously acted against them. In Sir Joshua Reynolds' diary, for instance, we read that in order to produce certain tints together. Now, orpiment is one of the colors of the second category, carmine-lake one of the third. That is to say: orpiment, as long as it remains isolated, keeps its brilliant yellow or reddish-orange color; but when mixed with white ic, and it, moreover, blackens the white lead, because the lated, does not stand as an oil color, and therefore has been superseded by madder-lake.

Unfortunately some of the most brilliant colors are per-
hable to such a degree that they ought never to be used; yet, it seems to me, that just in one branch of art in which of late remarkable progress has been made, I mean land color not easily to be realized, do not always resist the
cot temptation to make use of a number of pigments, the non-
durability of which is proved beyond doubt. However that may be, I think it pretty certain that the pigments in themselves play only a subordinate part in the deterioration of
oil paintings, and that the principal part belongs to the vehicle with which the colors are ground, and to the liquids
which are added during the painting. I bope, therefore, you will excuse my

\section{PHOTOGRAPH ENLARGEMENTS}

Trrs invention, by Messrs. Warlich \& Cadett, of England, has for its object the production of enlarged photographs
direct from negative or positive pictures. A condenser is placed at the proper distance from the
light to condense the rays thereof, the said condenser being

Fig. 1.

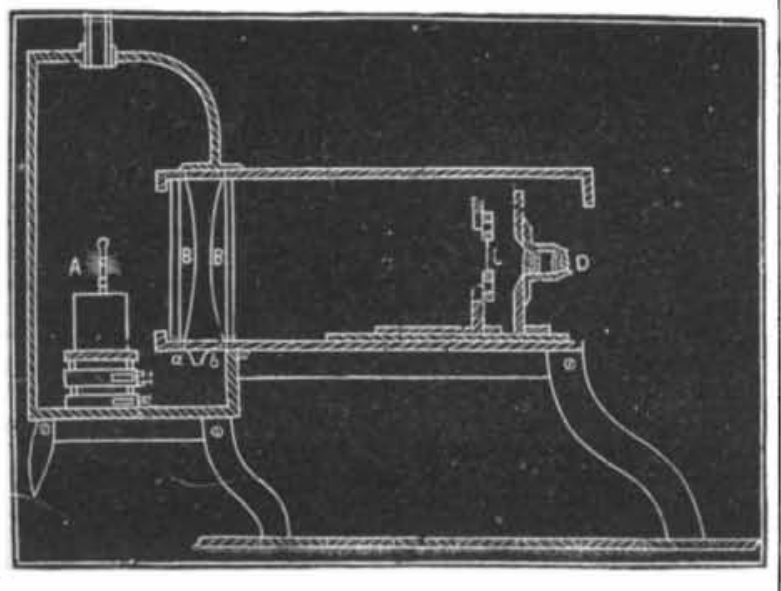

bollow, so as to allow of being kept filled with water or other liquid, and by means of an arrangement of pipes be considered fit for the purpose is made constantly or at in of keeping it cool.

The apparatus which holds the said condenser bas also similar arrangement, by which liquid can be made to flo
through it for the purpose of keeping it cool.

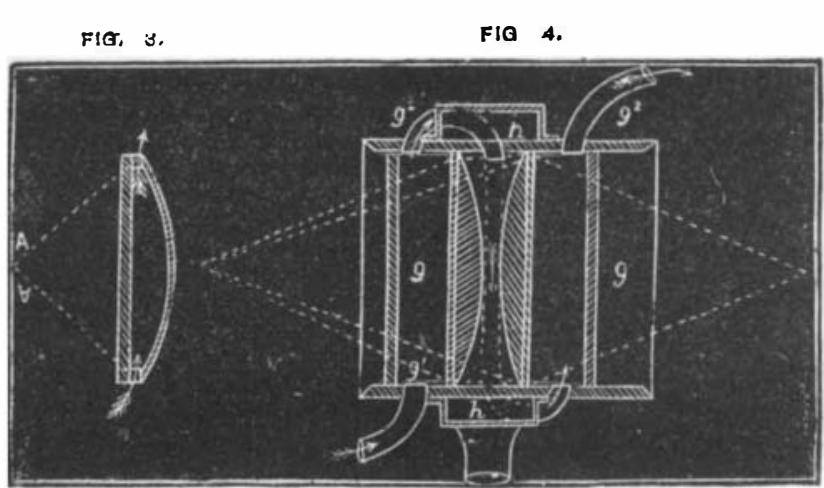

Glass condensers, in place of the bollow conclensers just or means would rise and fall upon $s^{1}$, and contact would be described, can be used for the purpose of this invention, but made by expansion of $s^{1}$ through beat, and communication in such case it would be desirable to interpose between the
light and glass condenser a glass trough or transparent re-
detent would thus be released, and the lamp raised until the ceptacle for water or transparent liquid, having arrange- light assumed its normalposition between $s$ and $s^{1}$, as shown.
ments for changing or flowing the water or liquid through If the light be too high the focal point would fall and act the same. Such receptacle for flowing the water or other upon $s$, and, by the electrical communication, the magnet, liquid can be formed by making one face of the lens a side
for such receptacle, a plate of glass forming the other side
, would act upon the detent and lower the lamp.
In Fig. 3 the water or other fluid which circulates between, ject to be treated. from $A$, and such rays are condensed and pass through the negative or positive picture, $C$, to be enlarged, and are
then taken up by the lens or lenses, $D$, and projected at the proper focus to produce the enlarged image at $E$ from are, as before described, hollow, to allow a continuous flow of water or liquid through the same, the water or liquid thence by the pipe or other means conveyed down and into

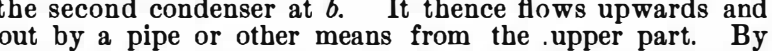
this continuous flow of water or liquid through the condensers the same are kept cool and insure the efficient lenses, and such arrangements are for keeping the light
lon In any part within the path of the rays is placed a lens, $b^{2}$, are springs or means, $s, s^{1}$, which by the effect of the heat communication the lens make contact with the magnets, $m, m^{\prime}$, act upon detents which free clockwork or is raised or lowered to keep the light central with the con-
ingers

The action is as follows: Suppore the light falls too low,

FIO 4. partment. transparent receptacle might be used, in which the picture ept circulating through the trough or receptacle, or is condenser, from which emanate the condensed ras the light, and at a proper distance from the said condenser, is placed the positive or negative picture to be enlarged, and
at a still greater and proper distance in the same line a lens Tie condensed rays of light, on leaving the said condenser. De negative or positive picture to be enlarged, are then takn up by the above lens, the result bis, and an image of the above positive or negative picture is proproject upon any suitable surface sensitive to light. When the electric light is used an arrangement is, or may employed for keeping the light central with the said con-
enser and lens, which it does by means of clockwork or

FIQ 2.

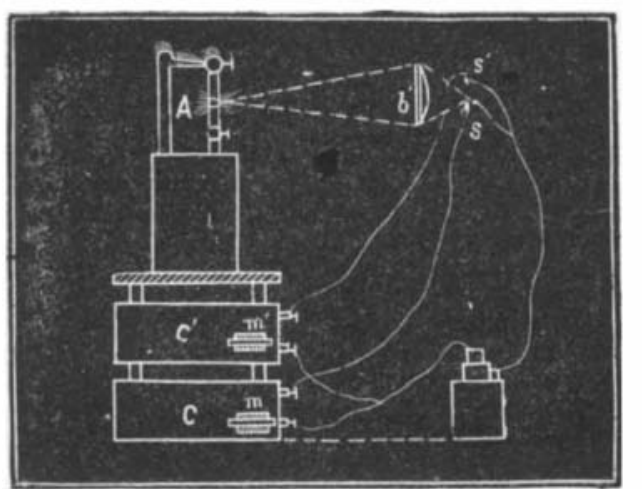

wise, and utilizing part of the heat evolved from the In using sunlight the box or lantern used with artificial the said condenser by the usual means.

Fig. 1 shows in outline the arrangements for producing Fig. 2 is a view in outline of arrangements for keeping the Fig. 3 shows a form of hollow lens.

wite

Fig. 5 is a front view of Fig. 4.

ptacle placed in front of the lensep. $A$ is the point where the light is placed; $B$, the condenlers; C, position of negative or positive picture; D, lens or
lenses placed parallel with the condensers, B; E, position for enlarged negative.
It is to be understood that the drawing is simply for the purpose of exemplifying the method adopted, as the positions larged image depend entirely upon the operator and the subThe arrangements shown in Fig. 2 are placed so that they 